\title{
DISSECTING ANEURYSM OF THE AORTA: A NEW SIGN
}

\author{
BY \\ J. A. NISSIM \\ From the North Staffordshire Royal Infirmary \\ Received September 2, 1946
}

Ever since Shennan's (1934) masterly and comprehensive analysis of 317 cases of dissecting aneurysm of the aorta, including 17 of his own, interest in this condition has been steadily increasing, with the encouraging result of a mounting proportion of antemortem diagnosis. Shennan reported only 6 such correct diagnoses out of the 317 cases, up to and including 1932, and credited Swaine (1855-6) with being the first to make it. Reich (1944) reported a further total of 153 American cases since Shennan, 36 of which were diagnosed: he added 19 cases of his own but omitted Logue's (1943) remarkable series of 12 cases, of which 10 were correctly diagnosed. Thomas (1945) reported another case, also diagnosed during life. This alone brings the total up to 49 correct diagnoses out of 512 collected cases. The incidence of this disease as given by Shennan is one in every 175 autopsies. Reich in 1944 worked out the average incidence given by various writers as one in every 380 autopsies.

The effect of the stimulus Shennan provided has been kept alive by subsequent writings. This has resulted in a general change of attitude, to use the words of Glendy et al. (1937), "from one of remote academic interest in the pathological aspects of the subject to one of lively practical attention to the possibility of making an ante-mortem diagnosis." Apart from the description of new signs, the features that should provide a lead to the diagnosis have been amplified. The following case is recorded on account of its many interesting features. Diagnosis during life was expressed in these words: "Theoretically, the only diagnosis that would fit all the symptoms and signs of the patient is dissecting aneurysm of the aorta." In view of a suspicious peptic ulcer history during the previous year, however, and the presence of abdominal signs with release tenderness etc., an abdominal emergency, superimposed on a background of cardiovascular disease, could not be ruled out. Further, a sign, not hitherto mentioned in published writings, which promises to be of pathognomonic significance when present, will be described.

\section{CASE RePORT}

A man of 71 years of age was sent into hospital with a short note from his doctor stating that the patient had a sudden attack of severe pain in his stomach the same day, and was thought to have a perforation of the stomach: $1 / 4$ of a grain of morphine sulphate had been given.

Seen by the resident surgical officer, the diagnosis of perforated peptic ulcer did not seem conclusive. Interrogation revealed that the patient was reclining in an armchair that morning, when suddenly he felt an extremely sharp and severe pain, a little below the umbilicus, soon spreading to the epigastrium and lower part of the chest, round both sides to the back, but not elsewhere. There was no retrosternal pain, and no nausea or vomiting. Slight dyspnœa was complained of. There was no disturbance of bowel movements, which had been regular up to and including that morning. For the past year he had some pain suggestive of peptic ulcer, which sometimes woke him up in the night and was often relieved by taking food. He also gave a history of high blood pressure for some time, and typical anginal pain, which he had experienced on four or five occasions; the pains came on with exertion and disappeared fairly quickly with rest.

On examination he showed an anxious pale face, and was obviously in severe pain, but could move about in bed. His temperature was subnormal and his respiration about 20 a minute. The abdomen was found to move well with respiration, but there was tenderness and guarding all over. Release tenderness was elicited as well, especially from the epigastric and right hypochondriac regions. There was no shifting dullness, no palpable abdominal mass, and no abnormal sounds on auscultation. 
The heart was not obviously enlarged. A radiogram of the chest, however, taken the next day about two hours before death, showed a rather enlarged heart shadow with a widened supracardiac shadow. The heart sounds were faint but regular. There was persistent bradycardia of 44 beats a minute, but the previous rate was unknown. No murmurs or other adventitious sounds could be heard. Examination of the lungs, the central nervous system, and the urine yielded no abnormal findings.

Some condition simulating coronary thrombosis that could explain these signs and symptoms was sought and dissecting aneurysm of the aorta seemed a possibility. The radial pulses on the two sides were found different in strength and confirmation was obtained by the sphygmomanometric reading of of $80 /$ ? on the right and $120 / ?$ on the left. The dorsalis pedis and posterior tibial arteries were easily felt in both legs and found of equal volume. A slight transient " peculiar" feeling in his head was later mentioned by the patient to have preceded the onset of the severe pain in the infra-umbilical region by a short while.

Another finding that came to notice later was a difference between the right and left carotid pulsations. They were reduplicated on the right and single on the left, without a coexisting noticeable difference in the intensity of the pulsations on the two sides. There were two almost equal beats in quick succession on the right carotid for every single beat on the left. The right radial pulse, however, like the left, was single but weaker. Electrocardiography could not be carried out owing to an unfortunate breakdown of the apparatus.

The same evening, the pulse rate dropped to 36 , although his general condition was improved. The next day it went up to about 55 a minute. Towards the evening the patient developed an acute exacerbation of the symptoms and of the abdominal signs-excruciating epigastric pain radiating below the umbilicus and upwards into the lower chest, with guarding that almost amounted to rigidity. About an hour later he gave a few gasping breaths and died suddenly.

Necropsy (Dr. A. J. McCall). The body was that of a rather emaciated man.

Skull and brain. These were not examined.

Thorax. There was a hæmopericardium with $600 \mathrm{~g}$. of recent blood clot closely investing the heart. The aorta showed a $2.5 \mathrm{~cm}$. horizontal tear about $4 \mathrm{~cm}$. above the aortic ring. At right angles to this and running into it was a longitudinal tear, which had curled round beneath the adventitia. In one place, the adventitia had given way, giving rise to the hæmopericardium. Blood had spread beneath the epicardium and into the auricular septum where hæmorrhages were present in close relation to the A-V bundle. Distally, the blood had dissected the aorta down to the fourth lumbar vertebra, where a second tear allowed re-entry into the aorta. Dissection had occurred along the innominate artery, but not along the left subclavian or left carotid arteries, so that the difference in blood pressure during life on the two sides was explained. The aorta at the site of the rupture was thinned but not otherwise obviously abnormal. Distally, however, there was much atheroma and some pearly intimal thickening.

Heart. This was not unduly large. The coronary arteries showed only slight atheroma.

Abdomen. Some blood had effused behind the peritoneum in front of the pancreas and in its substance. The spleen was slightly enlarged and firm. The abdominal organs were otherwise healthy.

\section{Discussion}

Clearly the terminal event causing the sudden death was the rupture into the pericardial sac, with a consequent hæmopericardium and tamponade. The partial heart block could be explained by the blood seeping into the auricular septum and across the A-V bundle, and the low abdominal pain by extension of the dissection as far down as the fourth lumbar vertebra, but why the pain was first manifest there was not clear. The severe epigastric pain simulating an acute abdominal emergency seems to be best explained by the hæmorrhage in front of and in the substance of the pancreas.

The only single diagnosis to explain the multiplicity of signs and symptoms seemed that of dissecting aneurysm of the aorta. In the absence of pathognomonic signs, however, the suspicious past history of the patient with a double retrospective diagnosis of peptic ulcer and angina pectoris, and the obvious abdominal signs including release tenderness, especially for the first few hours, combined to make the consideration of other conditions in the differential diagnosis seem necessary.

Cardiac infarction seemed unlikely and syphilitic aneurysm of the upper aorta could cause the pain in the lower chest and the difference in the brachial blood pressure, but other corroborative signs were missing. Acute abdominal conditions could not explain the brachial difference in blood pressure and the partial heart block, but it was thought that they might 
supervene in a case that had those signs already from preceding hypertension, arteriosclerosis, and myocardial degeneration. Not long before, a patient suspected of having aortic aneurysm because of a difference in brachial blood pressure of $30 \mathrm{~mm}$. was shown at necropsy to have bronchial carcinoma and a deformed right clavicle.

Even after dismissing the cardiac signs as coincidental, any of the abdominal conditions thought of seemed to leave a great deal unexplained. Mesenteric embolism lacked its usual abdominal distension, colicky obstructive type of pain, and nausea and vomiting. Acute pancreatitis was more seriously considered, but the onset seemed rather too sudden and still did not explain the initial low pain. Perforation of a peptic ulcer lacked its board-like rigidity and restriction of abdominal mobility with respiration. The subsidence of the abdominal pain without the supervention of a peritonitic stage at the time of re-examination by the surgeon seemed almost to rule it out.

It is interesting to note what Shennan wrote regarding the course of the pain. "In some cases a very characteristic feature which gives an important clue to the condition present is the gradual disappearance of the pain, the relief persists for hours or days or even a week or two, but the pain recurs later with similar rapidity and rapid death." Although the relief in this case was far from complete, the sequence of events seems to be very similar.

The clinical manifestations of dissecting aneurysm of the aorta are protean. Simple meditation on the anatomical aspects would soon conjure up an almost endless chain of symptoms, signs, and differential diagnoses. It is the coexistence of certain groups of signs and symptoms rather than any single one of them that should almost always lead one to suspect the condition. Sometimes, however, it is a long way from making certain of the diagnosis.

Among the leading symptoms of this catastrophe, the pain with its type, mode of onset, and distribution is of paramount importance in diagnosis. It is usually very severe, extremely sudden, and is described as splitting, tearing, rending, crushing, or knife-like in character. As a rule, it is felt in the midthorax, front or back, and usually descending from above downwards. It may be felt in the neck, mandible, head, beneath the left or right scapula, in the shoulders, lumbar regions, loins, epigastrium, right or left hypochondrium, right or left iliac fossa, and infra-umbilical region. It may also radiate into the legs, as far down as the toes, but radiation into the arms is unusual. Typical coronary thrombosis pain has also been described from extension of the dissection into the ostia of the coronary arteries. Sweating, faintness, shock, and collapse are frequent and may be extreme. Palpitation from extrasystoles or ventricular tachycardia may be complained of. Dyspnœa is frequently reported and appears to be more pronounced than is generally realized; it may be severe and lead to orthopnœa. Another important group of symptoms is due to the dissection extending along the peripheral vessels: along the external iliacs producing numbness, coldness, and weakness of the legs; along the coeliac axis or mesenteric vessels produeing nausea and vomiting, diarrhœa, hæmatemesis, melæna, abdominal cramps, or distension; or along the renal vessels, producing hæmaturia and anuria. Even subcutaneous hæmatomata have been recorded, from dissection of the superficial vessels. Involvement of the cerebral arteries can result in a whole gamut of symptoms from headache to coma: visual disturbances, facial paralysis, loss of speech, hemiplegia, paraplegia, visceral pains of tabetic type, and incontinence. A third variegated group may be met with from rupture of the dissecting aneurysm anywhere inside the chest, and from pressure effects so produced: cough, sensation of suffocation, dysphagia, hoarseness, and hæmoptysis. Symptomless cases are known to occur.

A sign has been met with in the present case to which no reference could be found. This was the unilateral reduplicated beat of the carotid artery. The explanation must lie in the difference of the rate of propagation of the pulse wave through the lumen of the artery and through its dissected coats where the blood was probably partly clotted. At necropsy, this was the only pathological finding that could account for it. The full significance of this sign was not realized until then. White (1944) in his authoritative summary of dissecting aneurysms says, "There are no pathognomonic signs of dissecting aneurysms." Since, however, there does not seem to be any other pathology that could produce this unilateral double beat in an artery, its pathognomonic significance is at once appreciated. The frequency and usefulness of this sign may be greater than is at present realized, and it may occur in other arteries. Looking for this sign by palpation of all accessible arteries may reveal further instances. 
Two other signs of dissecting aneurysm have been added in recent years. Roesler, Gifford, and Betts (1937) described the appearance of a rapidly shifting area of pulsation in the interscapular area, over which the aortic second sound was very accentuated: it was also associated with a rapid change in the radiological appearance of the aortic shadow. The other sign was due to Logue (1943) and consisted of a bruit and a thrill over the femoral artery.

Delay in the conduction of pulse beats is also significant. "In dissecting aneurysm of the aorta delay of the pulse beat, when compared with the apical beat, can occur in all arteries, resulting from delay and weakening of the blood stream entering or passing through the involved artery or arteries" (Shennan, 1934). Appreciable help may be obtained from the search for these simple signs in suspected cases.

\section{SUMMARY}

A review of the improved diagnosis of dissecting aneurysm of the aorta during life is presented. Its manifestations are protean, but some leading symptoms are singled out.

A case in which this diagnosis was considered as the only one to include all the signs and symptoms under a single pathology is described.

A new sign of this disease is described consisting of unilateral reduplicated arterial pulsation, in this case in the right carotid artery. Its pathognomonic significance is discussed, and other recently described signs are reviewed.

I should like to render my thanks to Dr. A. Wilson Gill for his help and Dr. A. J. McCall for the use of the post-mortem report.

\section{REFERENCES}

Glendy, R. E., Castleman, B., and White, P. D. (1937). Amer. Heart J., 13, 129.

Logue, R. B. (1943). Amer. J. med. Sci., 206, 54.

Reich, N. E. (1944). Clinics, 3, 346.

Roesler, H., Gifford, U. G., and Betts, W. (1937). Amer. Heart J., 13, 426.

Shennan, T. (1934). Dissecting Aneurysms, M.R.C. Report No. 193, H.M. Stationery Office, London.

Swaine (1856). Trans. Path. Soc., 7, 106.

Thomas, G. T. (1945). Clinical J., 74, 20.

White, P. D. (1944). " Heart Disease,' 3rd ed., Macmillan, New York. 